\title{
Variação sazonal da contaminação por helmintos em matrizes ovinas e borregos submetidos a controle integrado e criados em pastagens tropicais
}

\author{
Seasonal variation of helminthes contamination in ewes and lambs under integrated control \\ and raised in tropical pastures
}

\author{
Flávia Aparecida Nogueira' ${ }^{\mathrm{I}}$ Fabrício Teixeira da Rocha' ${ }^{\mathrm{I}}$ Gustavo Cardoso Ribeiro ${ }^{\mathrm{I}}$ \\ Nathalie Oliveira Silva ${ }^{I}$ Luciana Castro Geraseev ${ }^{I}$ Anna Christina de Almeida \\ Eduardo Robson Duarte*
}

\section{RESUMO}

A verminose é um dos principais problemas sanitários da criação de pequenos ruminantes no Brasil e no mundo. Entretanto, pouco se conhece sobre a variação sazonal da contaminação parasitária e os efeitos da associação de um controle estratégico com um tático sobre ovinos criados em condições do Semiárido no norte de Minas. Este trabalho foi realizado com o objetivo de avaliar a variação sazonal da contaminação por helmintos gastrintestinais em borregos $e$ matrizes ovinas em um sistema de controle integrado no norte de Minas Gerais, Brasil. Durante um ano, mensalmente, foram coletadas amostras de 30 borregos e 30 matrizes de uma propriedade. Durante o mês de abril de 2007, foram também avaliados 68 ovinos em uma segunda propriedade, período esse que correspondeu à época de parição nas duas fazendas. Amostras de fezes foram obtidas diretamente da ampola retal para determinação do número de ovos por gramas de fezes $(O P G)$ e os dados submetidos à análise de variância. Os valores médios do OPG das matrizes apresentaram aumento significativo nos meses de maio e outubro de 2007, meses de parição na propriedade. Já para os borregos, ocorreu aumento significativo no OPG médio em março de 2007, coincidindo com o final da estação chuvosa. O controle integrado adotado foi eficiente na redução da contaminação após esses períodos relatados. Após o cultivo das larvas, os gêneros mais observados foram Trichostrongylus, Haemonchus e Strongyloides, tanto para o período seco, quanto para o chuvoso. A comparação das contaminações das matrizes das duas propriedades no mês de abril de 2007 indicou uma média de OPG significativamente maior na segunda propriedade, que não tratou esses animais antes do parto.

Palavras-chave: epidemiologia, helmintose ovina, periparto, controle integrado.

\begin{abstract}
The verminosis is one of the principal sanitary problems in ovine herds in Brazil and around the world. However, little is known about the seasonal variation of the helminth contamination and the effects of the combination of a strategy control and a tactical control in sheep raised under in the Northern Minas semiarid conditions. In this study, the objective was to evaluate the seasonal helminth contaminations in lambs and ewes under an integrated control in the North of Minas Gerais, Brazil. The research was carried through during December 2006 to November 2007. Monthly faecal samples from 30 lambs and 30 ewes of one farm were collected. During April 2007, 68 sheep of a second farm were also evaluated, corresponding to the peri-partum time to both farms. The samples were collected directly from the recto and the faecal egg count (FEC) was determined and evaluated by variance analysis. The ewe FEC increased significantly in May and October, which were peri-partum periods. Lamb FEC increased significantly in March, which coincided with the rainy season end. The integrated control was efficient to reduce the contaminations after these related periods. After the larvae culture, the genus Trichostrongylus, Haemonchus and Strongyloides were the most observed to both dry and rainy periods. During April 2007 the ewe contamination was significantly higher in the second farm that did not treat these animals in the pre-partum.
\end{abstract}

Key words: epidemiology, ovine helminthosis, peri-partum, integrated control.

\section{INTRODUÇÃO}

O norte de Minas Gerais possui clima e vegetação favoráveis à espécie ovina e apresenta

Instituto de Ciências Agrárias, Universidade Federal de Minas Gerais (UFMG), 39400-006, Montes Claros, MG, Brasil. E-mail: duartevet@hotmail.com.*Autor para correspondência. 
grande potencial para se tornar um importante polo produtor desses animais. O rebanho ovino dessa região atualmente é de aproximadamente 554.103 animais (ANUALPEC, 2006). Entretanto, os sistemas de criação predominantes são caracterizados por baixos índices zootécnicos, em conseqüência da precária nutrição, dos problemas sanitários, do manejo ineficiente e do baixo potencial genético dos animais (GERASEEV et al., 2007).

A redução de produtividade e o aumento da mortalidade estão relacionados diretamente à ocorrência de verminose nesses animais. Em um estudo realizado no Estado de São Paulo, os prejuízos são mais acentuados no período de inverno e no início da primavera, associados ao periparto e às condições precárias de alimentação, comuns nessa época (AMARANTE, 1993).

A baixa resistência de ruminantes jovens aos endoparasitas tem sido verificada e está associada a uma menor resposta imunológica contra esses parasitos. Um agravante a essa situação é que, durante o periparto, as matrizes eliminam mais ovos de nematóides nas fezes, aumentando a contaminação das pastagens. Tem-se observado que o OPG em animais jovens aumenta quando esses animais são criados juntos com as mães (COLDITZ et al., 1996).

Os fatores ambientais influenciam diretamente a composição e a regulação da população parasitária (STROMBERG, 1997), interferindo principalmente sobre os estádios larvais no pasto e determinando também a predominância de uma ou mais espécies em regiões específicas (BEVERIDGE et al., 1989). Em países de clima tropical, a temperatura e a umidade são os fatores mais importantes, sendo responsáveis pelo desenvolvimento de ovos e larvas no ambiente, durante grande período do ano (VALCARCEL et al., 1999).

Pouco se conhece sobre a epidemiologia das helmintoses em ovinos criados sob condições do semiárido tropical e os efeitos de um controle integrado na contaminação de ovinos de diferentes categorias. Sendo assim, o objetivo neste estudo foi avaliar e comparar a variação sazonal da contaminação por helmintos gastrintestinais em borregos e em matrizes ovinas criados em pastagem tropical, em região semiárida, e submetidos a um controle estratégico e tático.

\section{MATERIAIS E MÉTODOS}

Caracterização da área

O trabalho foi conduzido no período de dezembro de 2006 a novembro de 2007, em uma propriedade com área total de 126ha, sendo que 60ha eram divididos em seis piquetes com tamanho de 2 a 10ha cada, destinados a 143 matrizes ovinas. A propriedade localiza-se na cidade de Francisco Sá, inserida na mesorregião do norte do Estado de Minas Gerais, Brasil. A cidade está localizada a $16^{\circ} 44^{\prime} 13,25^{\prime}$ ” de latitude Sul, 4351'53,06” de longitude Leste de Greenwich e a $465 \mathrm{~m}$ de altitude.

O clima da região é caracterizado como tropical típico, quente e semiúmido, com uma estação chuvosa curta (verão) e outra seca longa (inverno) bem definida. Este clima é caracterizado como Aw, ou seja, tropical quente, com chuvas de verão e temperaturas médias anuais entre 22 e $24^{\circ} \mathrm{C}$ e pluviosidade média anual entre 1000 e $1200 \mathrm{~mm}$ (KOPPEN, 1948).

\section{Manejo e caracterização dos animais}

Na propriedade, eram adotadas práticas de manejo integrado, como a vermifugação no pré-parto, em que as matrizes eram tratadas e separadas em piquetes-maternidade. Já os borregos seguiam o esquema de vermifugação estratégica, sendo tratados no fim de março, na segunda semana de julho, de setembro e de novembro. Os animais foram tratados com moxidectina na dose de $2 \mathrm{mg} \mathrm{kg}^{-1}$ de peso corporal, utilizando-se a via oral. A eficácia desse vermífugo foi avaliada dois meses antes do início do experimento de acordo com os procedimentos descritos em COLES et al. (1992). Foi observada redução de 92\% no OPG das matrizes tratadas com essa base, demonstrando sua eficácia.

Os borregos permaneciam junto de suas mães nas pastagens, eram alimentados separadamente no aprisco, eram desmamados com três a quatro meses de idade e então criados em piquetes separados das matrizes. Nessa propriedade, foram adotadas duas estações de monta, sendo que as parições ocorreram nos meses de abril a junho e de outubro a novembro. Os percentuais de parição foram de 86 e 68\% para o início e o final do período seco, respectivamente. Durante um ano, a cada mês, foram avaliados 30 borregos mestiços de dois a seis meses de idade e 30 matrizes mestiças Santa Inês adultas no periparto (30 dias antes ou até 60 dias pós-parto).

Durante o mês de abril de 2007, em uma segunda propriedade da mesma mesorregião, com um rebanho com as mesmas características anteriores, foram também coletadas fezes de 28 matrizes e 44 borregos. Esse mês correspondeu à época de parição nas duas fazendas, sendo que, na segunda propriedade, não se administrou anti-helmíntico às matrizes no pré-parto, não as separando em piquetematernidade. 
Exames parasitológicos

Para a contagem de OPG, foram coletadas 5 a 15 gramas de fezes, diretamente da ampola retal, com sacos plásticos que foram acondicionados em caixas isotérmicas e transportados para o laboratório de parasitologia do Instituto de Ciências Agrárias/UFMG. As coletas foram sempre realizadas nos últimos dias de cada mês avaliado, respeitando-se o período mínimo 15 dias após a última vermifugação. As análises foram realizadas utilizando-se solução saturada de cloreto de sódio e leitura no microscópio em duas câmaras de McMaster (WHITLOCK 1948) para cada amostra , obtendo-se o valor do OPG médio para cada animal.

A identificação dos principais gêneros presentes nos animais avaliados nos meses de julho e dezembro foi realizada após a visualização microscópica de larvas de terceiro estágio. Os cultivos das amostras proveniente de 60 matrizes, nesses dois meses, e a identificação das larvas obtidas foram realizados segundo o método e as características descritas em UENO \& GONÇALVES (1998) e KEITH(1953).

\section{Análise estatística}

Para análise estática, os dados obtidos sofreram transformação logarítmica Log10 (X+1), sendo submetidos à análise de variância em um sistema fatorial 2X12. As médias foram comparadas pelo teste ScottKnott, considerando-se o nível de significância de 5\%.
A diferença na prevalência dos gêneros de L3 obtidas no período seco e no chuvoso do ano foi avaliada pelo teste do qui-quadrado.

\section{RESULTADOS E DISCUSSÃO}

A análise da contaminação por trichostrongilídeos de matrizes e borregos ao longo do ano mostrou que a média geral do OPG das matrizes (419 \pm 1814 ) foi significativamente maior $\mathrm{P}<0,05$ ), quando comparada à dos borregos (166 \pm 631$)$. Esses resultados indicam que mesmo com um controle tático para as verminoses, os animais adultos mantiveram maiores taxas de contaminação, podendo ser considerados reservatórios de populações de nematoides e contribuindo como fonte de infecção para o rebanho. Os dados climáticos da região avaliada indicaram médias para temperatura e umidade relativa do ar de $23,4^{\circ} \mathrm{C}$ e $60,6 \%$, respectivamente. A pluviosidade nessa região foi de $755,4 \mathrm{~mm}$; entretanto, durante o período de março a setembro, a precipitação mensal foi inferior a $17 \mathrm{~mm}$ ou foi nula, reduzindo a umidade nos meses subsequentes e caracterizando a condição climática semiárida e tropical (Figura 1).

Os meses de maio e outubro foram os períodos com maiores médias de OPG para as matrizes e corresponderam aos meses de parição (Figura 1, $\mathrm{P}<0,001)$. Os mecanismos de influência do periparto

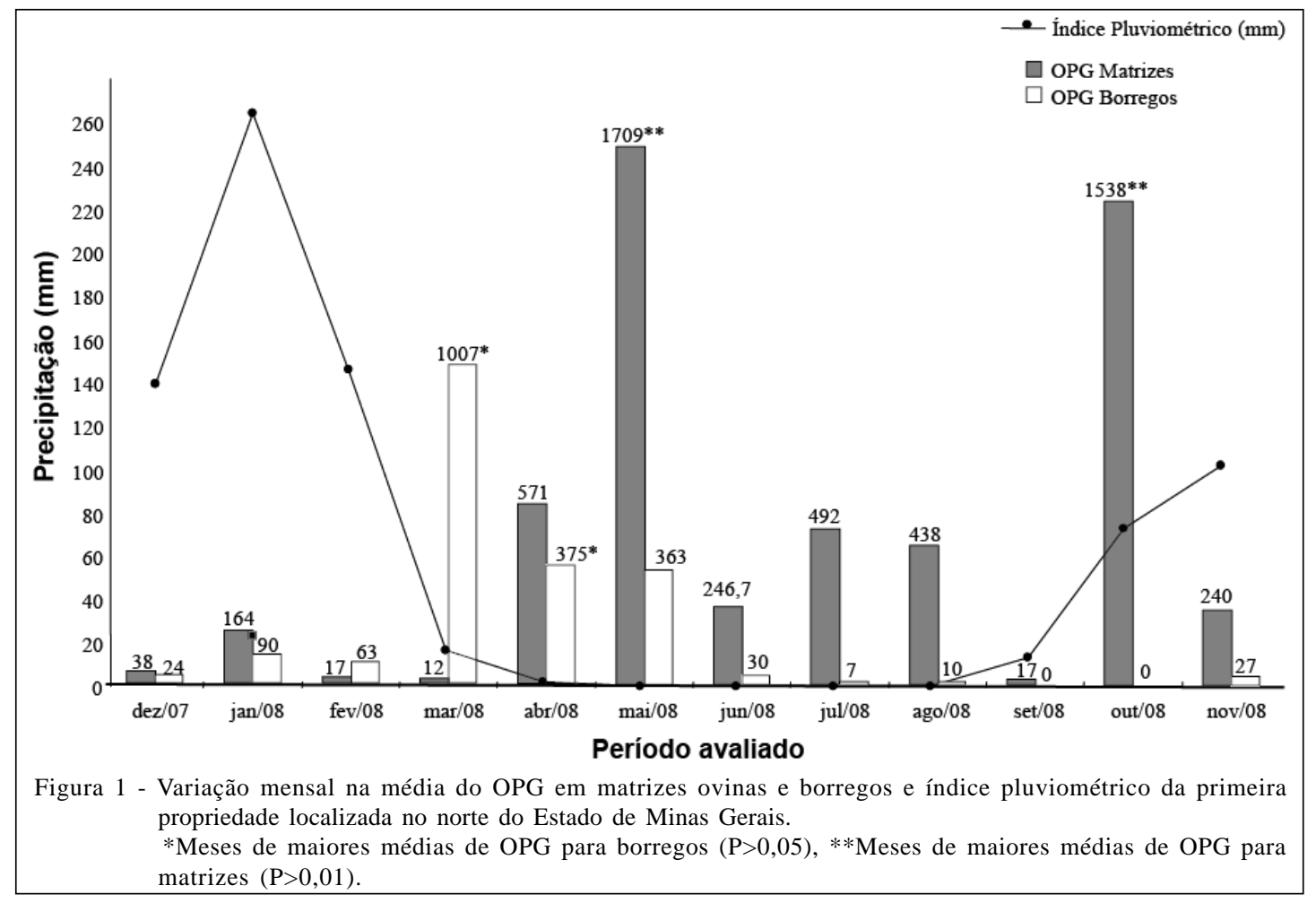

Ciência Rural, v.39, n.9, dez, 2009. 
são ainda pouco elucidados na literatura científica, porém acredita-se que sejam provocados por imunodepressão de origem endócrina, decorrente de variações hormonais que ocorrem próximas ao parto e durante a lactação(SOULSBY,1987; GREER, 2008). Em um estudo, FLEMING (1993) observou que, em ovelhas submetidas às doses diárias de prolactina no período de trinta dias de infecção, houve um aumento expressivo no OPG em relação ao grupo controle, demonstrando que animais no periparto, sob influência desse hormônio, poderiam aumentar o OPG.

Após o parto, com o decorrer da lactação, a imunidade retornaria à normalidade, ocasionando a redução de larvas, que completam seu ciclo parasitário, e diminuindo o OPG (JANSEN, 1987). Portanto, os aumentos no OPG, observados em maio de 2007 e outubro de 2007, podem estar relacionados à depressão do sistema imune das ovelhas, resultando, possivelmente, no aumento do número de larvas que atingiram os estádios reprodutivos. Entretanto, logo após esse período, houve queda significativa no OPG devido possivelmente à eficácia da vermifugação no pré-parto (Figura 1).

Em um estudo da contaminação de ovelhas Santa Inês no periparto, criadas em pastagem de Brachiaria brizantha, no pantanal brasileiro, foi observado que o período dos 10 primeiros dias pósparto foi o mais crítico para o aumento do OPG. A vermifugação dos animais no periparto, 10 dias antes do parto ou até nos primeiros 10 dias após o parto, constitui uma prática de tratamento tático adequada e recomendada, já que os animais diminuirão a carga parasitária e terão melhores condições corporais para o período de parto e início da lactação (SASA et al., 2008). Em outro estudo, foi também observado que o OPG de ovelhas da raça Merino Australiano no periparto pode chegar a 2.891, enquanto que, em outros períodos, o grau de infecção alcança uma média de 354 OPG (AMARANTE, 1993). A maior susceptibilidade de ovelhas no periparto às infecções por nematódeos gastrintestinais tem sido atribuída também ao aumento da exigência nutricional nessa fase, e o nutriente mais limitante, relacionado a essa queda da imunidade, tem sido a energia (HOUDIJK, 2008).

Os meses com maiores médias de OPG para os borregos foram respectivamente março, abril e janeiro (Figura 1, $\mathrm{P}<0,05$ ). Esse período correspondeu ao fim do período chuvoso da região, época de maior umidade relativa do ar e de temperaturas elevadas, que favoreceram o desenvolvimento de larvas infectantes. A menor eliminação de ovos pelos nematoides de borregos ocorreu durante o período seco, indicando que o controle estratégico adotado foi eficiente para reduzir a infecção nesses animais (Figura 1). Esses resultados estão de acordo com os de LIMA (1998), que observou níveis de infecção mais altos para bovinos de corte durante o período chuvoso. Nessa época, o desenvolvimento dos estádios de vida livre e a migração das larvas infectantes do bolo fecal para as pastagens adjacentes são favorecidos pelas condições climáticas.

Os principais gêneros identificados após os cultivos foram Trichostrongylus, Haemonchus e Strongyloides, tanto para o período seco, quanto para o chuvoso (Tabela1). A frequência desses gêneros de larvas para ambos os períodos foram semelhantes ( $\mathrm{P}>0,05)$.

Durante o mês de abril, a média do OPG (5272) das fêmeas da segunda propriedade foi significativamente maior ( $\mathrm{p}<0,001$ ), quando comparada com a da primeira propriedade e a dos borregos nas duas propriedades (Figura 2). Nesse plantel, o proprietário e os tratadores relataram a mortalidade de 35\% das matrizes durante a estação de parição avaliada. Esse fato pode estar relacionado às práticas de manejo adotadas em cada propriedade. Na primeira, as fêmeas foram vermifugadas 20 dias antes do parto e mantidas em piquetes-maternidade, enquanto que, na segunda, essas práticas não foram realizadas. Aumentos expressivos no OPG de fêmeas não vermifugadas no periparto têm sido relatados também nos estudos de AMARANTE, (1993), AMARANTE \& BARBOSA (1995) e SASA et al., (2008). Pelos dados obtidos nessas pesquisas, somados aos resultados deste estudo, verifica-se a importância de um controle tático da helmintose, enfatizando-se a vermifugação obrigatória das matrizes no pré-parto. Essa prática, além de minimizar as perdas econômicas e de produtividade, seria primordial para se manter a saúde e a vida das matrizes em rebanhos ovinos criados em condições tropicais.

Tabela 1 - Porcentagem de larvas de helmintos recuperadas dos cultivos de larvas provenientes de matrizes ovinas criadas no norte de Minas Gerais, durante os meses de julho e dezembro de 2007.

\begin{tabular}{lcc}
\hline Principais gêneros & julho (\%) & dezembro (\%) \\
\hline Trichostrongylus & 51,4 & 50 \\
Haemonchus & 26,4 & 30,9 \\
Cooperia & 3,2 & 0 \\
Strongyloides & 19,0 & 19,1 \\
\hline
\end{tabular}




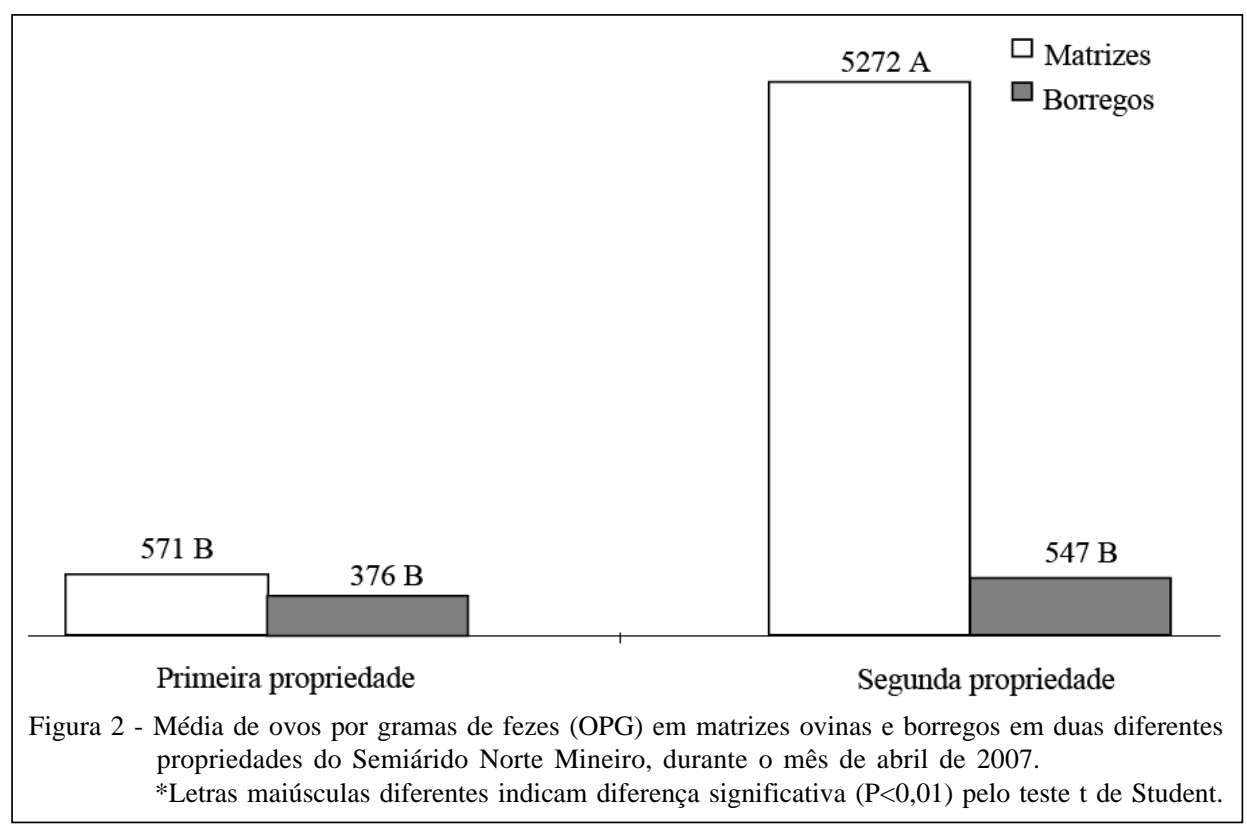

\section{CONCLUSÕES}

Os resultados indicaram que, para as matrizes, independentemente da variação climática ao longo do ano, os meses de maior eliminação de ovos de nematoides corresponderam ao período de periparto. O controle tático no pré-parto foi essencial para se reduzir a contaminação após as duas estações de parições avaliadas. Para os borregos, a época de maior eliminação de ovos correspondeu ao fim do período chuvoso e o controle estratégico foi eficiente para reduzir e manter o OPG em níveis baixos durante todo o período de seca, para a região avaliada.

\section{AGRADECIMENTOS}

Ao Fundo de Desenvolvimento Científico e Tecnológico - FUNDECI/Banco do Nordeste do Brasil; à Fundação de Amparo à Pesquisa do Estado de Minas Gerais FAPEMIG - ao Instituto de Ciências Agrárias da Universidade Federal de Minas Gerais - ICA/UFMG - e aos proprietários e trabalhadores rurais que contribuíram para este estudo.

\section{COMITÊ DE ÉTICA E BIOSSEGURANÇA}

Os procedimentos adotados com os animais neste trabalho estiveram de acordo com os princípios éticos da experimentação animal, sendo aprovados no protocolo 42/ 2008, pelo Comitê de Ética em Experimentação Animal da UFMG.

\section{REFERÊNCIAS}

AMARANTE, A.F.T.; BARBOSA, M.A. Seasonal variation in populations of infective larvae on pasture and nematode feacal egg output in sheep. Veterinária e Zootecnia, v.7, p.127133, 1995.

AMARANTE, A.F.T. Eliminação de ovos de nematódeos gastrintestinais por ovelhas de quatro raças durante diferentes fases reprodutivas. Pesquisa Agropecuária Brasileira, v.27, n.1, p.161-172, 1993. Disponível em: <http://webnotes.sct.embrapa.br/ pab/pab.nsf/FrAnual>. Acesso em: 10 ago. 2009.

ANUALPEC. Anuário da pecuária brasileira. São Paulo, 2006. 369p.

BEVERIDGE, I. et al. Effects of temperature and relative humidity on development an survival of the free-living stages of Trichostrongylus colubriformis, T. rugatus and T. virinus. Veterinary Parasitology, v.33, n.2, p.143-153, 1989. Disponível em: <www.sciencedirect.com/ science?_ob=MImg\&_imagekey=B6TD7-476MR83-YR$1 \&$ \&cdi $=5191 \&$ \&user $=10 \&$ \&orig $=$ search \&_coverDate $=09$ \% 2 F $30 \% 2$ F $1989 \&$ sk = 999669997 \& vi e w $=\mathrm{c} \& \mathrm{w} \mathrm{c} \mathrm{h} \mathrm{p}=\mathrm{d}$ G L b V S k W b \& m d $5=$ e b 52 a 848ed5346d9c0949bc332113a98\&ie=/sdarticle.pdf >. Acesso em: 09 ago. 2009. doi:10.1016/0304-4017(89)90062-9.

COLES, G.C. et al. World association for the advancement of veterinary parasitology (WAAVP) methods for the detection of anthelmintic resistance in nematodes of veterinary importance. Veterinary Parasitology, v.44, n.1, p.35-44, 1992. Disponível em: <http://www.sciencedirect.com/ science?_ob=MImg\&_imagekey=B6TD7-476MR61-XN\&_ c d i $=5191$ \& _ u s e r $=10$ \& _ o r i g $=$

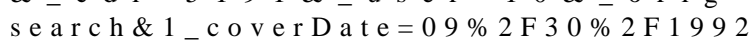
\& sk $=999559998$ \& vi e w $=c$ \& w chp $=$ d G LbVzb Skzk\&md5=18ec85a272be0917f2a499793972d048\&ie=/ sdarticle.pdf $>$. Acesso em: 08 de ago. 2009. doi:10.1016/ 0304-4017(92)90141-U.

COLDITZ, I.G. et al. Some relationships between age, immune responsiveness and resistance to parasites in ruminants. International Journal for Parasitology, v.26, n.1, p.869877, 1996. Disponível em: <http://www.sciencedirect.com/

Ciência Rural, v.39, n.9, dez, 2009. 
science?_ob=ArticleURL\&_udi=B6T7F-488XGV89\&_user $=10 \&$ \&coverDate $=09 \% 2 F 30 \% 2 F 1996 \&$ alid $=977410540$

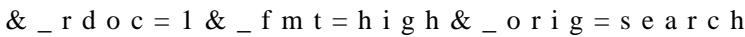
\&_c di=5 057 \&_sort=r\&_doc anchor=\&vie w $=\mathrm{c} \&$ \& $\mathrm{ct}=1 \&$ \&_acct $=\mathrm{C} 000050221 \&$ _version $=1 \&$ \& urlV ersion=0\&_userid=10\&md5=a3dfcfedc8e611e2f8e11981a8402dcf $>$. Acesso em: 8 ago. 2009. doi:10.1016/S0020-7519(96)80058-0.

FLEMING, M.W. Acute or chronic administration of prolactin alters ovine infection of Haemonchus contortus. Veterinary Parasitology, v.50, n.1, p.109-115, 1993. Disponível em: <http:/ /www.sciencedirect.com/science?_ob=MImg\&_imagekey=B6TD7$476 \mathrm{MNVN}-7 \mathrm{Y}-1 \&$ \&_cdi $=5191 \&$ \&user $=10 \&$ \&_orig $=$ searc h \& _ c o v e r D a t e = 1 $0 \% 2$ F $31 \% 2$ F 1993 \&_s k = 999499998\&vie w = c \& w c h p =d G L z V l z zSkzS\&md5=7bb850dd0582e3f6e10aecebdc029853\&ie=/ sdarticle.pdf>. Acesso em: 10 ago. 2009. doi:10.1016/03044017(93)90011-B.

GERASEEV, L.C. et al. Diagnóstico e recomendações técnicas para ovino caprinocultura no Norte de Minas. Montes Claros: Instituto de Ciências Agrárias da UFMG, 2007. 40p.

GREER, A.W. Trade-offs and bene fits: implications of promoting a strong immunity to gastrointestinal parasites in sheep. Parasite Immunology, v.30, n.1, p.123-132, 2008. Disponível em: <http://www3.interscience.wiley.com/search/ allsearch ?mode $=$ citation \& contextLink=blah\&issn=1365$3024 \&$ volume $=30 \&$ issue $=\&$ pages $=123-132>$. Acesso em: 08 ago. 2009. doi: 10.1111/j.1365-3024.2008.00998.x.

HOUDIJK, J.G.M. Influence of periparturient nutritional demand on resistance to parasites in livestock. Parasite Immunology, v.30, n. 1, p.113-121, 2008. Disponível em: $<\mathrm{ht} \mathrm{tp://www3.interscience.wiley.com/search/}$ allsearch mode $=$ citation \&contextLink=blah\&issn=13653024 \&volume $=30 \&$ issue $=$ \&pages $=113-121>$. Acesso em: 09 ago. 2009. doi: 10.1111/j.1365-3024.2008.00992.x.

JANSEN, J. The peri-parturient rise in sheep: feacal worm egg counts in normal and late lambing ewes. Veterinary Quarterly, v.9, n.2, p.97-102, 1987.

KEITH, R.K. The differentiation of the infective larvae of some common nematode. Australian, Journal of Zoology, v.1, n.1, p.223-235, 1953. Disponível em: <http://www.publish.csiro.au/ nid/90/paper/ZO9530223.htm>. Acesso em: 07 ago. 2009. doi: 10.1071/ZO9530223.

KOPPEN, W. Climatologia. Buenos Aires: Panamericana, 1948. 478p.

LIMA, W.S. Seasonal infection pattern of gastrointestina nematodes of beef cattle in Minas Gerais state-Brazil. Veterinary Parasitology, v.74, n.2, p.203-214,1998. Disponível em: <http:/
/ $\mathrm{w}$ w w. s c i e $\mathrm{n}$ c e d i r e c t. c o m/ science?_ob=MImg\&_imagekey=B6TD7-3SR342P-D$1 \&$ _ c d i $=5191 \&$ \& u s e r $=10 \&$ _ o r i g = s e a r c h \& c o v e r D a t e = $01 \% 2$ F $31 \% 2$ F $1998 \&$ \& sk=999259997\& \&iew $=$ c \& w chp $=$ dG L z Vtz zSkWz\&md5=f1aff593eefe $38467444 \mathrm{fb} 9 \mathrm{f} 19 \mathrm{f} 473 \mathrm{ca} \& \mathrm{ie}=$ sdarticle.pdf $>$. Acesso em: 06 ago. 2009. doi:10.1016/S03044017(97)00164-7.

SASA, A. et al. Infecção helmíntica em ovelhas Santa Inês no periparto criadas na região do Pantanal brasileiro. Revista Brasileira Saúde e Produção Animal, v.9, n.1, p.321-326, 2008. Miranda, MS. Disponível em: <http://revistas.ufba.br/ index.php/rbspa/article/view/889/629>. Acesso em: 10 ago. 2009.

SOULSBY, E.J.L. The evasium of the immune response and immunological unreponsivenes: parasitc helminthes infection. Immunology Letters, v.16, n.1, p.315-320,1987. Disponível em: $<\mathrm{ht} \mathrm{t} \mathrm{p} \mathrm{:} \mathrm{/} \mathrm{/} \mathrm{w} \mathrm{w} \mathrm{w.} \mathrm{s} \mathrm{c} \mathrm{i} \mathrm{e} \mathrm{n} \mathrm{c} \mathrm{ed} \mathrm{i} \mathrm{r} \mathrm{e} \mathrm{c} \mathrm{t} \mathrm{c} \mathrm{o} \mathrm{m} \mathrm{/}$ science?_ob=ArticleURL\&_udi=B6T 75-476HMX5S\&_user=10\&_coverDate $=12 \% 2 F 31 \% 2 F 1987 \&$ alid $=977406697 \&$

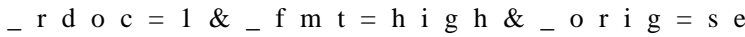
a r c h \&_c d i $=5049 \&$ s ort $=$ r \&_d o c a n c h o $\mathrm{r}=\& \mathrm{view}=\mathrm{c} \&$ \& $\mathrm{ct}=1 \&$ \&_acct $=\mathrm{C} 000050221 \&$ \& version $=1 \&$ _urlVersion=0\&_userid=10\&md5=7dbb4d4f9cdeb4f04a81b1196ba78f71>. Acesso em: 06 ago. 2009. doi:10.1016/0165-2478(87)90164-7.

STROMBERG, B.E. Environmental factors influencing transmission. Veterinary Parasitology, v.72, n.1. p.247264, 1997. Disponível em: <http://www.sciencedirect.com/ science?_ob=MImg\&_imagekey=B6TD7-3S53W11-3$1 \&$ \&di $=5191 \&$ \&user $=10 \&$ \& orig $=$ search \&_coverDate $=$ 11\%2F30\%2F1997\&_sk=999279996\&view=c\&wchp=dGLbVlzzSkWz\&md5=0ff99365307fffe2c3f58ceb654f1937\&ie=/ sdarticle.pdf $>$. Acesso em: 05 ago. 2008. doi:10.1016/S03044017(97)00100-3.

UENO, H.; GONÇALVES, P.C. Manual para diagnóstico das helmintoses de ruminantes. 4.ed. Tokyo: Japan Internatinal Cooperation Agency, 1998. 143p.

VALCARCEL, F. et al. Prevalence and seasonal pattern caprine Trichostrongyles in a dry area central Spain. Journal Veterinary Medicine Series B, v.46, p.673-681, 1999. Disponível em: <http://www.unboundmedicine.com/medline/ebm/record/ 10676144/abstract/Prevalence_and_seasonal_pattern_of_capri ne_trichostrongyles_in_a_dry_area_of_central_Spain_>. Acesso em: 06 ago. 2009. doi.wiley.com/10.1046/j.14390450.1999.00297.x.

WHITLOCK, H.V Some modifications of the McMáster helminth egg-counting technique and apparatus. Journal of Council for Scientific and Industrial Research, v.21, p.177-180, 1948. 